\title{
Heart Anomaly Detection using Deep Learning Approach Supported Signal Analysis
}

\author{
Prof. Himanshu Joshi ${ }^{1}$ \\ ${ }^{1}$ Professor of Computer Engineering \& Savitribai Phule Pune \\ University, JSPM'S Imperial College of Engineering \& \\ Research Wagholi, Pune, India
}

\author{
Vaibhav Salunke ${ }^{2}$, Pranav Dhabale ${ }^{3}$, Parikshit Yalawar ${ }^{4}$, \\ Kamlesh Vidhate ${ }^{5}$ \\ 2,3,4,5 Pursuing Bachelor of Computer Engineering Savitribai \\ Phule Pune University, JSPM'S Imperial College of \\ Engineering \& Research Wagholi, Pune, India
}

\begin{abstract}
Phonocardiography is an effective technique Which helps for the recording of heart sounds during the heart cycle Identification and further diagnosis of human related diseases Heart. Contraction of the heart muscles and closure of the heart valve the heart produces sound, which can be analysed by the experienced Cardiologist. The aim of this study is to create an automated classification method using a one-dimensional solution Neural network based on phonocardiogram data for discrepancies in the heart sound.
\end{abstract}

The proposed system consists of three phases.

1) Data Acquisition 2) Pre-Process

2) 3) Feature Extraction and Classification.

We have proposed an intelligent neural network approach for the classification of PCG data. Heart sound recording (PCG data) Which is nothing but a audio file converted in its time Domain representation. This is provided as converted PCG data input for the Neural Network. Was also emphasized on Noisy heart sound recording. It Can reduce noise efficiency Classification because it disrupts the values of the neural network. The accuracy of the proposed system is $91.5 \%$ with sensitivity of 0.92 and specificity 0.91.

Keywords -Convolution neural network, PCG data, phonocardiogram, heart sound.

\section{INTRODUCTION}

According to the World Health Organization (WHO), cardiovascular disease (CVD) is the most common cause. Death globally. CVD kills more people than any other disease in the world. More than 17.5 million people worldwide have died Is due to cardiovascular diseases. About 610,000 people Every year in the United States die of heart disease is the same More than $29 \%$ of total deaths in 1 in 4 deaths 2004 is due to cardiovascular diseases and is number one Is increasing every day. Coronary heart disease (CHD) is the most common type of heart disease, 37 kills more than 370,000 People annually. Existing methods of finding the primary Signs of abnormalities in the heart are very expensive. They may not be Where it is cheaper in underdeveloped and developing

countries the economic condition of the country is not so good. So, their A possible and reliable system is needed for early detection Heart disorders. Any method that can help you find it Signs of heart disease can therefore have a significant effect on world health.

A stethoscope is an acoustic medical device for isolation or for listening to the internal sounds of the human body. That is A primary device for hearing the heart sound. The advantage of This is to use an electronic stethoscope over an acoustic stethoscope Its properties like amplified sound output, enhanced frequency

Range, ambient noise reduction, etc. It includes an amplifier to amplify low intensity heart sound. Electronic stethoscopes transmit sound electronically, therefore, it can be wireless

Can be a device or a recording device. It can also provide a scene Performance of recorded heart sound.

PCG recordings have four heart sound signals

I.e. S1, S2, S3, S4. The first two are normal heart sounds Normal heart valves are produced by opening. S3 and S3 have unusual heart sounds like S1 S2 and S4. These unusual sounds are called rumblings. Current Medical testing techniques that can detect the deformity

Heart sounds are very expensive. It is not affordable for the average human being. So, the main challenge is to develop such Accurate, reliable and affordable technology.

Heart sound is still the primary tool for detection and Analysis of the condition of the human heart. Proper interpretation of the state of the heart is largely based on experience Cardiologists. It can be fraught with error. More reliable Computer based technology needs to be developed.

Several methods are being suggested for the medical system Development for the diagnosis of heart disease. The purpose is ours the study aims to propose an intelligent algorithm to determine the presence of abnormalities in the sound of patient data. Also, with this we wanted to make it possible and affordable Solution.

\section{METHODOLOGY}

The proposed method [1] in this study is the basic three Step Architecture 1) Data Acquisition 2) Pre-Processing 3) Classification

\subsection{Data Acquisition and Data Preparation}

Use of data sets for supervised machine learning purposes Pascal is a data set that includes the sound of the heart Artifact, recordings labelled by three categories of common name Heart sounds and abnormal heart sounds. About $400 \mathrm{HS}$ files There is in the Pascal data set.

In the processing step, we made two identical copies of Data set. In the first copy of a data set, labels are common and the unusual is replaced by a non-artifact label. This is done for signal quality 
assessment. Analogs are converted after HS In the domain introduction of his time [2]. The advantage of Converting analogue data to a time domain is Analogs the data is converted into a statistical equivalent representation which A machine is an understandable structure and it is also easy for the purpose of analysis. There can be various mathematical functions Analysis which applies to statistics and. Can be used for So time domain introduction is very important.

The input size of CNN is already fixed. So, the recordings Some have to be converted to a certain length before training [3]. We converted the signal into fixed size data of 8-11 seconds.

If it is longer than the specified time, we cut the data in fixed size. If it is short, we extend it by repeating the original signal to make it to the original length. Down sampling is applied to omit ineffective data. Down sampling improves normalization on data sets. Is there Some really low frequency noise recorded electronically Stethoscope, especially the rumble that passes through one Low pass filter that allows low frequency sound to pass through and Finally reject the sound from the data. So, this is the first A step towards filtering out noisy data.

\subsection{Signal pre-processing:}

The signal is pre-processed for quality assessment Heart sound files. The artwork contains sound files sound with data that is weak with quality. The H.S. The signs are not suitable for classification.

\subsection{Feature Extraction and Classification}

The signal quality assessment block [4] ensures that these files shresentation and understanding We have selected for 2 neurons be deleted. Now the data set is only with good quality audio duo ifiptesad.

Which is potentially fit for classification.

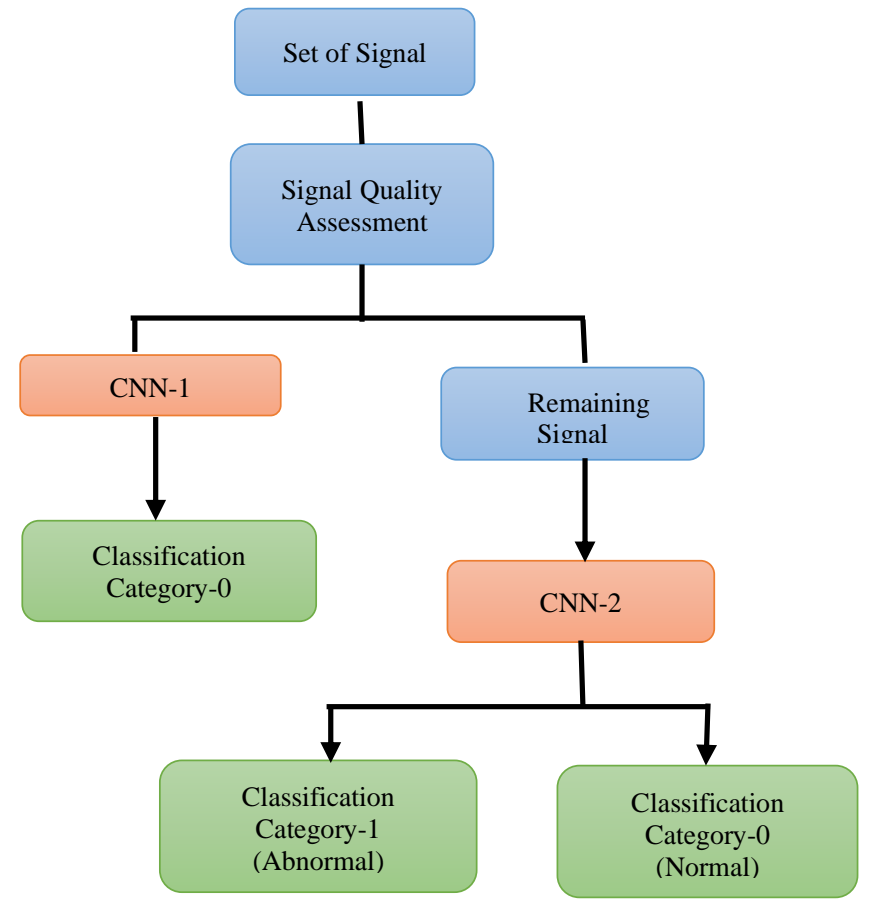

Fig -1: Proposed block diagram
CNN can be useful for classification and can be used Classified as good or bad quality [5]. So, here are the advantages Neural networks are exploited for signal quality assessment Classify heart sound files into good quality files Free from noise and poor-quality files that contain artifacts. The heart sound data set is given to the Convolution neural network Which classifies data into two categories called artifacts and non-artifact. The main reason for doing this is to prevent Values of neurons due to distortion. While training Neural network, values of neurons in each age $\mathrm{CNN}$ comes closer and closer to the real value and therefore functionality of the features presented on the neural network Grows slowly. So, if we delete the recordings in them Artifacts, we can increase the efficiency of classification Neural networks are therefore also known as CNN-1, good or bad Classifying a quality signal can also be seen as a first step Towards increasing the accuracy of the system. Greater Accuracy of CNN-1 to better filter poor quality signal There will be a demonstration of $\mathrm{CNN}-2$ for general and classification There will be an unusual heart sound and therefore better performance of the system.

The Convolution neural network is mainly composed of two Parts, feature extraction and classification. Section of Feature extraction is responsible for automating effective features from PCG signals. Classification Part of it uses the feature of reacted. In short, these two the departments carry out the main work of this paper in a cooperative manner.

For binary classification, the last layer has 2 neurons Shown. It can also be done using 1 neuron, but only for the better 


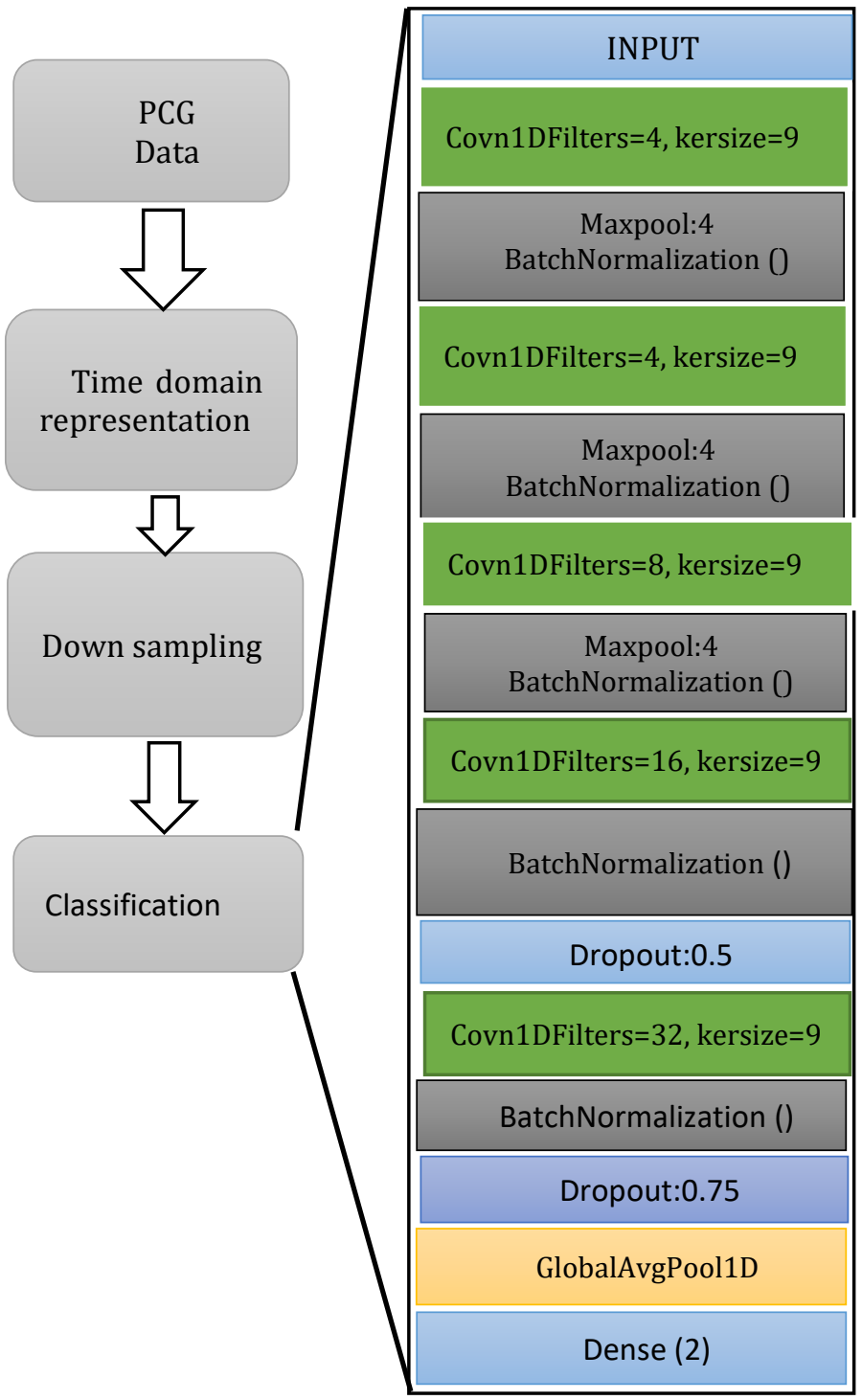

Fig -2: Proposed processing methodology
Batch normalization allows each level of the nerve A network for learning a little more independently Another level. It reduces over-fitting and increases stability Neural network.

2) Batch normalization: -: Batch normalization allows everyone The level of the neural network for learning a little more independently than the second level. It reduces and increases over-fitting Stability of the nerve network.

\begin{tabular}{|c|c|c|}
\hline Layer (type) & Output Shape & Param \# \\
\hline conv1d_ 7 (Conv1D) & (None, 1543, 4) & 40 \\
\hline max_pooling1d_5 (MaxPoølingl 1 & (None, 386, 4) & $\theta$ \\
\hline batch_normalization_ 7 (Batch & (None, 386, 4) & 16 \\
\hline conv1d_8 (Conv1D) & (None, 378, 4) & 148 \\
\hline max_pooling1d_6 (MaxPoøling1 & (None, 95,4 ) & a \\
\hline batch_normalization_8 (Batch & (None, 95,4 ) & 16 \\
\hline conv1d 9 (Conv1D) & (None, 87, 8) & 296 \\
\hline max_pooling1d_7 (MaxPooling1 & (None, 22, 8) & $\theta$ \\
\hline batch_normalization_9 (Bateh & (None, 22, 8) & 32 \\
\hline conv1d_10 (Conv1D) & (None, 14, 16) & 1168 \\
\hline max_pooling1d_8 (MaxPooling1 & (None, 4,16 ) & a \\
\hline batch_normalization_10 (Batc & (None, 4,16 ) & 64 \\
\hline dropout_4 (Dropout) & (None, 4,16 ) & $\theta$ \\
\hline conv1d 11 (Conv1D) & (None, 1, 64) & 4160 \\
\hline batch_normalization_l1 (Bate & (None, 1,64 ) & 256 \\
\hline dropout_5 (Dropout) & (None, 1, 64) & $\theta$ \\
\hline conv1d_12 (Conv1D) & (None, 1,32 ) & 2080 \\
\hline batch_normalization_12 (Batce & (None, 1, 32) & 128 \\
\hline dropout_6 (Dropout) & (None, 1,32 ) & $\theta$ \\
\hline global_average_pooling1d_z ( & (None, 32) & $\theta$ \\
\hline dense_ 2 (Dense) & $=$ & $===$ \\
\hline
\end{tabular}

Fig 3: Parameters of neural network

\section{RESULTS:}

General and absolute accuracy of the classification system Abnormal heart sound is $91.5 \%$. The accuracy of a good or bad quality classifier or $\mathrm{CNN}-1$ that is responsible for the signal.

The following purpose in the implementation of the neural netwo

Left: PCG data (.wav file) is converted to binary data Fixed length using time domain representation which is next Bored of the CNN model. Right: CNN Architecture includes a lot Convulsion and maximum pooling levels, batch normalization levels Fully connected or followed by dense layers.

\subsection{Dropout layer [6]: -}

The term "dropout" means leaving units (both hidden)And visible) in the neural network. That's the decent thing to do, and it should end therePerformance of the average model with a neural network. Model the average is a natural response to the uncertainty of the model. This The dropout level allows regularization by random setting Zero some neurons in the previous layers during training

1) Max Pooling [6]: -: Max Pooling is intended Input presentation for down-sample. It helps reduce Eliminates dimensionality and convenience extraction. It reduces Computing cost by reducing the number of parameters To learn.
The quality assessment is $86.7 \%$.

\section{Result}

Filename: murmur 201101051104.wav

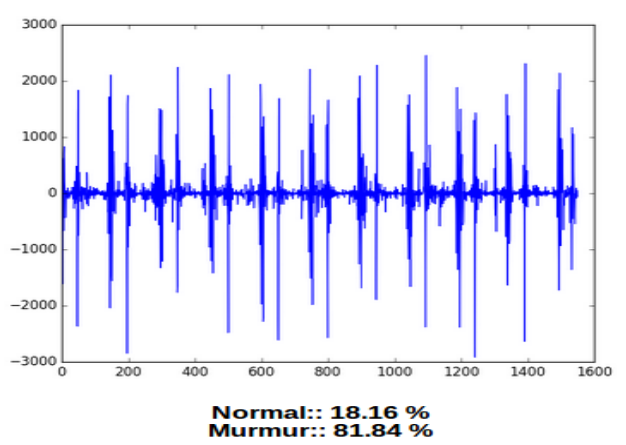




\section{Result}

Filename: normal_201102201230.wav

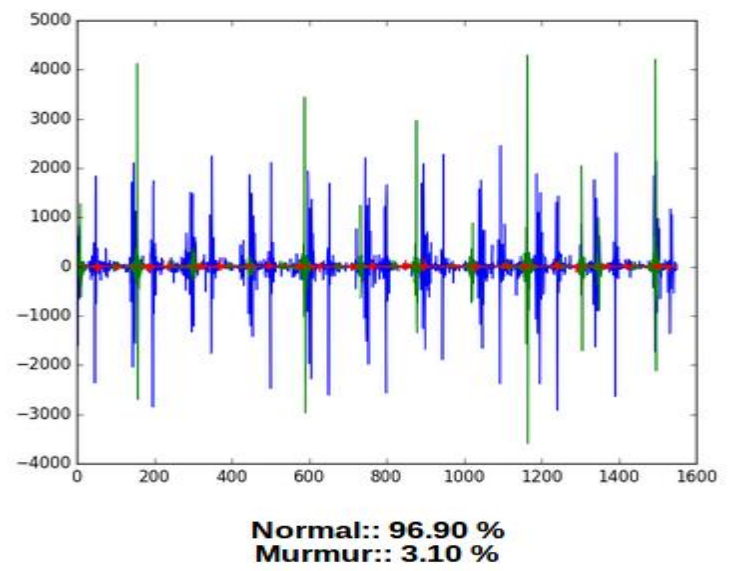

Result

Filename:: artifact_201106040933.wav

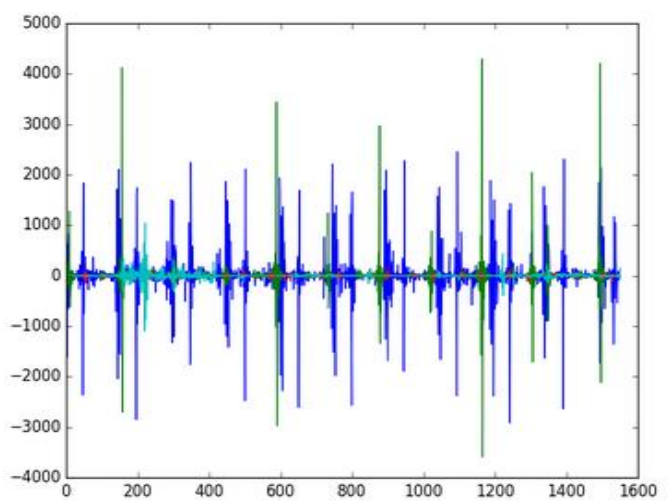

\section{REFERENCES}

[1] A. S. Y. M. N.-A. El-Sayed A. El-Dahshan, "Heart disease diagnosis using an intelligent algorithm based on PCG signal analysis." [Online]. Available: Jul.28,2017

[2] V. V. Nivitha and R. K. I., "Effective heart sound segmentation and murmur classification using empirical wavelet transform and instantaneous phase for an electronic stethoscope," IEEE Sensors Journal, vol. 17, no. 12, pp. 3861-3872, June 2017. [Online]. Available: 10.1109/JSEN.2017.2694970

[3] C. Schölzel and A. Dominik, "Can electrocardiogram classification be applied to phonocardiogram data? \#x2014; an analysis using recurrent neural networks," in 2016 Computing in Cardiology Conference (CinC), Sept 2016, pp. 581-584. [Online]. Available: 10.23919/CIC.2016. 7868809

[4] I. Grzegorczyk, M. Solinski, M., A. Perka, J. Rosi' ski, J. Rymko,' K. St1n, and J. Giera, "Pcg classification using a neural network' approach," in 2016 Computing in Cardiology Conference (CinC), Sept 2016, pp. 1129-1132. [Online]. Available: 10.23919/CIC.2016.7868946

[5] M. Zabihi, R. A. B., S. Kiranyaz, M. Gabbouj, and K. A. K., "Heart sound anomaly and quality detection using an ensemble of neural networks without segmentation," in 2016 Computing in Cardiology Conference (CinC), Sept 2016, pp. 613-616. [Online]. Available: 10.23919/CIC.2016.7868817

[6] P. Garg, E. Davenport, G. Murugesan, B. Wagner, C. Whitlow, J. Maldjian, and A. Montillo, "Automatic 1d convolutional neural-networkbased detection of artifacts in meg acquired without electrooculography or electrocardiography," in 2017 International Workshop on Pattern Recognition in Neuroimaging (PRNI), June 2017, pp. 1-4. [Online]. Available: 10.1109/PRNI.2017.7981506

[7] C. Potes, S. Parvaneh, A. Rahman, and B. Conroy, "Ensemble of featurebased and deep learning-based classifiers for detection of abnormal heart sounds," 2016 Computing in Cardiology Conference (CinC), Vancouver, BC, 2016, pp. 621-624.

[8] M. Gjoreski, A. Gradišek, B. Budna, M. Gams and G. Poglajen, "Machine Learning and End-to-End Deep Learning for the Detection of Chronic Heart Failure From Heart Sounds," in IEEE Access, vol. 8, pp. 20313-20324, 2020, DOI: 10.1109/ACCESS.2020.2968900.

[9] Todd R. Reed, Nancy E. Reed, Peter Fritzson, Heart sound analysis for symptom detection and computer-aided diagnosis, Simulation Modelling Practice, and Theory, Volume 12, Issue 2, 2004, Pages 129-146, ISSN 1569-190X, https://doi.org/10.1016/j.simpat.2003.11.005.

\section{CONCLUSION}

This study is very complex and attempts to solve the complex Problems in the medical field. It tries to classify the heart Sound is obtained by PCG in normal and abnormal hearts Sound. This will significantly help the health care industry the highest number of deaths worldwide is due to the heart Related problems. The method adopted for classification There is a technology in computer science known as neural network. The use of a one-dimensional neural network is demonstrated. The model proposed in this study demonstrates a novelty Approach to classification of noisy data containing artifacts. The first step is quality assessment. The advantage of this step Voiced data does not disturb the original neural A network that is used for the classification of good quality hearts Sound file in normal and abnormal heart sound. Instead, A separate neural network is used for the same purpose i.e. Classification of noisy heart sound data from good quality heart sound Data. The limitation is that the system will not produce significant Satisfactory results if the data set contains more files Noisy data. 